\title{
Synthesis and Preliminary Biological Studies of Novel Retinamide Derivatives
}

\author{
Hong S. Sin, ${ }^{*}$ Youn J. Kwon, Hye S. Han, ${ }^{\dagger}$ Si H. Park, Moong S. Park, Young S. Rho, ${ }^{\ddagger}$ and Soo J. Um ${ }^{* *}$ \\ Chebigen Inc., 305-B, Chungmugwan, Sejong Lniversitw, Seoul $1+3-747$, Korea \\ -Dept. of Bioscience \& Biotechnologv Institute of Bioscience, Sejong Lniversity, Seoul 143-747, Korea \\ -Dept of Chemistr, Chonbuk National Lniversitv, Choni $561-756$, Korea \\ Received Hav,+ 2002
}

\begin{abstract}
We have described the synthesis and biological activity of novel retinamide derivatives. The retinamide derivatives were synthesized by introducing functional side chains into the 4-hydroxy group of 4-HPR. The activities could be dependent on the side chain length. functional group. and hetero atom. The antiproliferative potential of the derivatives was assessed by MTT assay in HCT116 colon cancer cell lines.
\end{abstract}

Key Words : 4-HPR. Retinoid. Derivatives. Side chain. Biological studies

\section{Introduction}

Retinoids are natural and sy'nthetic analogues of vitamin $\mathrm{A}$ that are involved in the regulation of several biological functions such as cellular differentiation and proliferation. Clinically, retinoids are useful for the treatment of skin disorders and cancer ${ }^{\mathrm{l}}$ and are currently being investigated in several other therapeutic areas. including arthritis. ${ }^{2}$ dỵslipidemias. ${ }^{3}$ and the prevention of HIV induced ly'mphopenia. ${ }^{4}$

All-trons retinoic acid (RA) and its amide derivative. $N$-(4hỵdroxyphenyl)retinamide (4-HPR) were șinthesized years ago and found to be effective against various skin diseases and are now being considered as potential drugs for treatment and prevention of several cancers. ${ }^{5.6}$ Though 4 HPR is derived from the natural retinoid retinoic acid. it is less toxic and substantially less teratogenic than RA. Additionally, the lower toxicity of retinamide has led to its experimental use in animal studies as an antitumor agent ${ }^{8}$ and in clinical trials as a chemopreventive agent for breast cancer. ${ }^{9}$ 4-HPR displays antiproliferative effects in vitro against human breast carcinoma cells ${ }^{5}$ and induces apoptosis in the hemopoietic cell line..$^{10}$ A recent study suggests that 4 HPR is a highly selective activator of the retinoic acid receptor $\gamma$ (RAR $\gamma)^{9}$ inhibits AP-l activity. and induces apoptosis in ovarian cancer cells. ${ }^{11}$ Also it has been reported that some 4-HPR derivatives show greater efficacy and less toxicity than original $4-\mathrm{HPR}$ when tested in a culture of breast carcinoma cells. ${ }^{12}$ Furthermore. the chemopreventive potential of 4-HPR has been determined against the development and growth of 7,12-dimethylbenzanthracene (DMBA)-induced rat mammary tumor. ${ }^{13}$

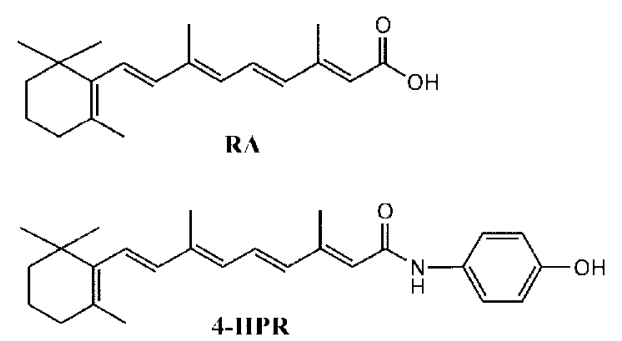

Figure 1.

\section{Results and Discussion}

In the present study. we describe the synthesis and biological activity of the retinamide derivatives. The derivatives were synthesized by introducing functional side chains into the 4-hydroxy group of HPR (Figure 2). The antiproliferative potential of the derivatives was determined by MTT in HCT 116 colon cancer cell lines.

Various conjugates of 4-HPR have been prepared in two steps as described in Schemes 1 and 2. The preparation of 4HPR derivatives (Figure 2) consisted of the general $O$ acylation of 4-HPR with various alkanoic acids. sulfonyl clilorides and phosphoryl chlorides.

As shown in Scheme 1, all-trans retinoic acid (RA) was converted into an acid chloride derivative using $\mathrm{SOCl}_{2}$ and dimethylformamide (DMF), and then coupled with $p$-aminophenol to obtain 4-HPR by a procedure recently described. ${ }^{14.15}$

The synthesis of retinamide derivatives was carried out as follows. First. derivatives $\mathbf{1 - 5}$ were prepared by the coupling of various alkanoic acids and 4-HPR under the conditions employing EDCI/DMAP at room temperature in dry DMF. $\alpha$-Keto form 1 and 2 were obtained in low yields, due to decomposition upon purification. Second, derivatives $\mathbf{6}$ and 7 were synthesized from the reaction of 4-HPR and alkane sulfonyl chlorides in presence of $N$-methylmorpholine (NMM) in dry $\mathrm{CH}_{2} \mathrm{Cl}_{2}$.

As in Scheme 2, treating the requisite alkyl alcohol with stoichiometric amount of phosphorous oxychloride under Ar in anlyydrous $\mathrm{CH}_{2} \mathrm{Cl}_{2}$ at reflux for $5 \mathrm{~h}$ provided the alkyl

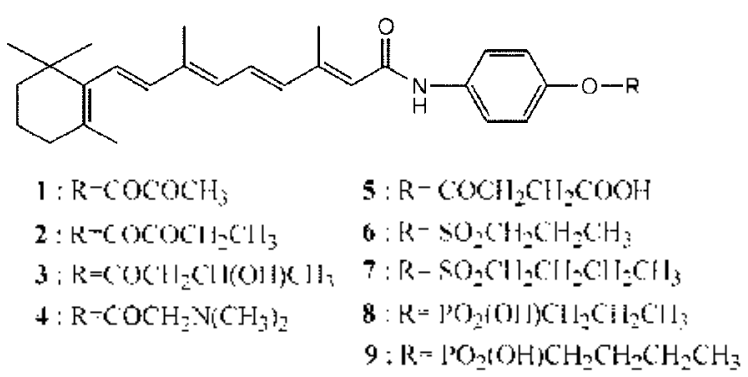

Figure 2. 


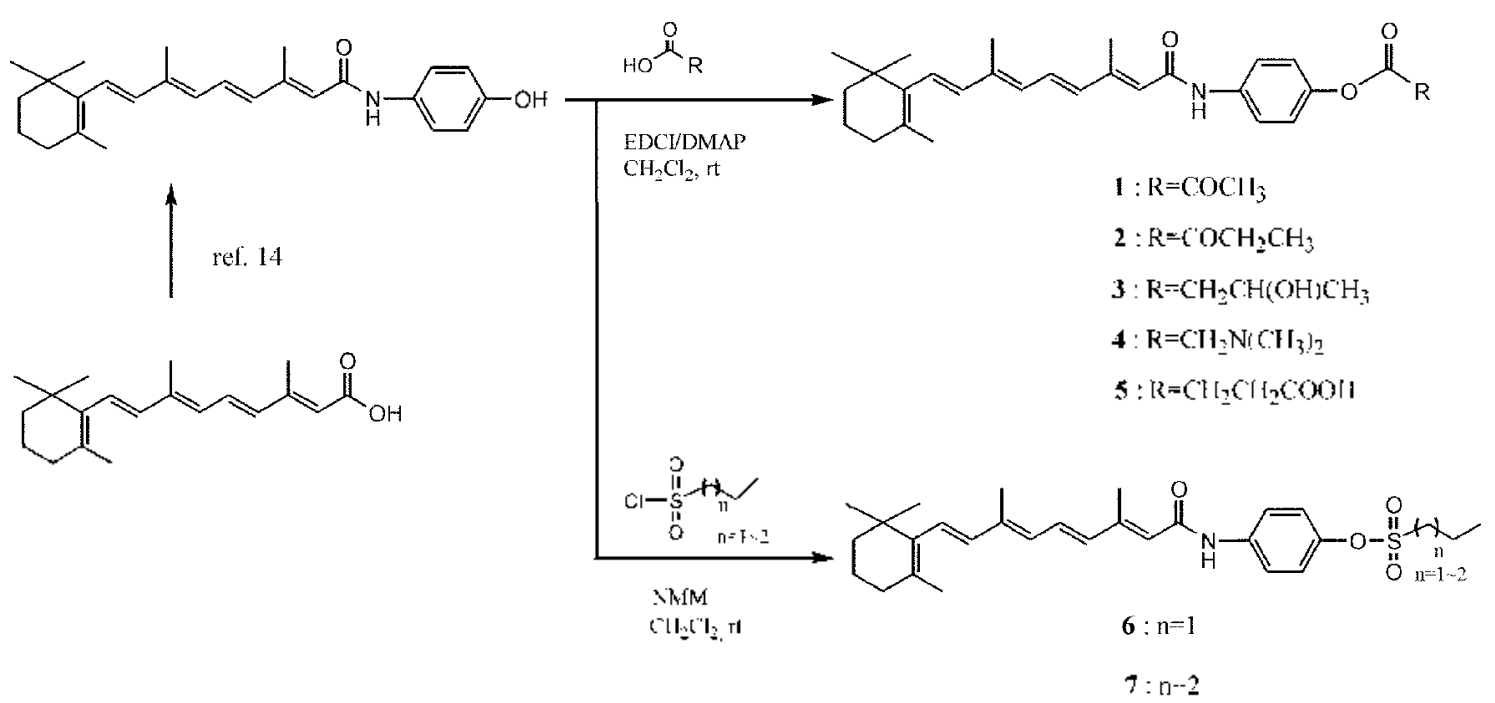

Scheme 1

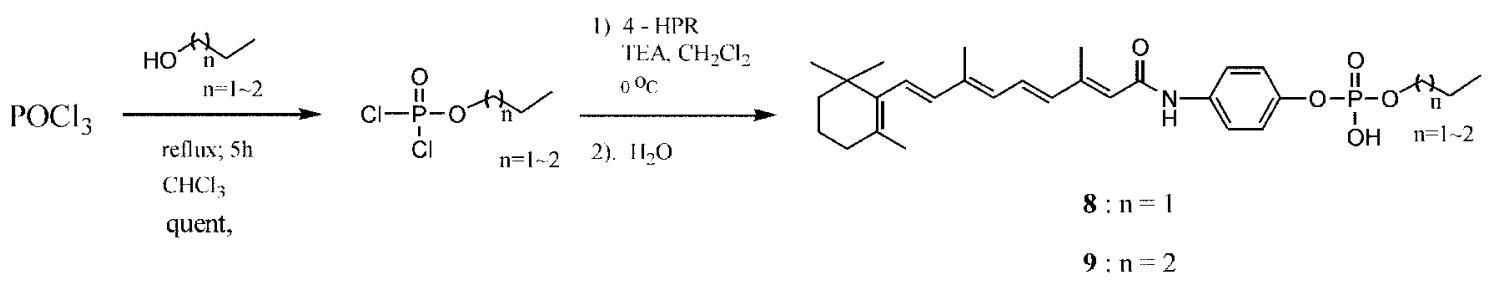

Scheme 2

dichlorophosphate in a quantitative vield. Derivatives 8 and 9 were prepared by phosphorylation of 4-HPR with alkyl dichlorophosphates in the presence of triethylamine at $0^{\circ} \mathrm{C}$ in dry $\mathrm{CH}_{2} \mathrm{Cl}_{2}$ and the lydrolysis of the corresponding adduct with $\mathrm{H}_{2} \mathrm{O}$ in good yields

Anti-proliferative effects of Retinamide derivatives. The growth suppressing potential of retinamide derivatives was investigated by deternining their $\mathrm{IC}_{\text {sij) }}$ values (concentrations giving 50\% growth inhibition) in HCT116 colon cancer cells. To determine the $\mathrm{IC}_{5 i}$ value. each derivative was treated at six different concentrations $(0,0.5 .1,2.5 .5$. $10 \mu \mathrm{M}$ ). As summarized in Table $1 . \mathrm{IC}_{\text {si }}$ of derivative 2 was $1.8 \mu \mathrm{M}$. four-fold better than 4-HPR $(7.0 \mu \mathrm{M})$ in HCT1 16 cells. $\mathrm{IC}_{\text {siv }}$ values of other derivatives were slightly better

Table 1. IC so values of retinamide derivatives in HCTI16 colon cancer cells

\begin{tabular}{cc}
\hline Derivatives & $\mathrm{IC}_{\text {s) }}(\mu \mathrm{M})$ \\
\hline +-HPR & 7.0 \\
$\mathbf{1}$ & 7.0 \\
$\mathbf{2}$ & 1.8 \\
$\mathbf{3}$ & 3.0 \\
$\mathbf{4}$ & 5.0 \\
$\mathbf{5}$ & 5.5 \\
$\mathbf{6}$ & 25.0 \\
$\mathbf{7}$ & 4.0 \\
$\mathbf{8}$ & $>100$ \\
$\mathbf{9}$ & $>100$ \\
\hline
\end{tabular}

than (derivatives $3,4,5.7$ ), similar to (derivative 1 ). or much worse than (derivatives $6,8,9$ ) that of 4 -HPR. For better clinical outcome. we searched for retinoid derivatives with $\mathrm{IC}_{5 i j}$ of submicromolar concentration and low side effects. Although the $\mathrm{IC}_{50}$ value of $4-\mathrm{HPR}$ is relatively high. its relatively few side effects made it useful in clinical trial. In this regard, our derivative 2 . the most effective agent among the derivatives tested, could be a promising cancer drug. although its side effects remain to be determined by animal studies.

\section{Experimental Section}

Dry DMF was stored over $4 \AA$ sieves and degassed before use by bubbling argon vigorously through for at least one hour. Dry $\mathrm{CH}_{2} \mathrm{Cl}_{2}$ was obtained from distillation over $\mathrm{CaH}_{2}$. Commercially available reagents and solvents were used without further purification. All reactions were conducted under an Ar atmosphere. except for those reactions utilizing water as a solvent. They were monitored by TLC (Merck Kieselgel $60 \mathrm{~F} 254$ ). All the products prepared were purified by flash column cluromatography using silica gel 60 (Merck. 230-400 mesh). ${ }^{1} \mathrm{H}$ NMR and ${ }^{13} \mathrm{C}$. NMR spectra were recorded on a Bruker AC-200F and JEOL JNM EX-400 using $\mathrm{CDCl}_{3}$ as the solvent. All chemical shifts $(\delta)$ are quoted in ppm downfield from TMS and coupling constants $(J)$ are given in $\mathrm{Hz}$. Mass spectra were measured on a Shimazu GCMS-PO 1000 mass spectrometer (EI $70 \mathrm{eV}$ ).

General procedure I; A preparation of 4-HPR. A 
mixture of dry DMF $(0.077 \mathrm{~mL} .0 .99 \mathrm{~mm}$ ol $)$ and $\mathrm{SOCl}$ $(0.072 \mathrm{~mL} .0 .99 \mathrm{mmol})$ was stirred under argon for $\mathrm{l} \mathrm{h}$. To the solution was added all-trans retinoic acid (100 ng, 0.33 mmol) in dry DMF ( $2 \mathrm{~mL}$ ). After being stirred at $0^{\circ} \mathrm{C}$ for 45 mintues in subdued light, the clear deep red retinoyl chloride solution was added dropwise to a cooled solution of distilled triethylamine $(0.14 \mathrm{~mL} .0 .99 \mathrm{mmol})$ and 4-aminophenol $(0.072$ g. $0.66 \mathrm{~mm}$ ol $)$ in dry. degassed DMF $(2 \mathrm{~mL})$. The temperature was maintained between $10-15$ degrees during the addition. The dark colored reaction mixture was stirred at room temperature until TLC analysis indicated no remaining 4-aminophenol (about $2 \mathrm{~h}$ ). The reaction was quenched with $\mathrm{NH}_{4} \mathrm{Cl}(a q$.$) and extracted with EtOAc. The extracts$ were washed with $\mathrm{H}_{3} \mathrm{O}$ and brine, dried $\left(\mathrm{Na}_{2} \mathrm{SO}_{4}\right)$, and concentration. The residue was purified by column chromatography using a hexane/EtOAc (3/1) as the eluent to give HPR (0.118 mg. $91 \%$ ) as a yellow solid.

General procedure II; 4 - $\{[(2 E, 4 E, 6 E, 8 E)-3,7$-Dimethyl9-(2,6,6-trimethyl-1-cyclohexenyl)-2,4,6,8-nonatetraenoyl]amino; phenyl-2-0xobutanoate (1). To a solution of EDCI (97.7 mg. $0.510 \mathrm{mmol})$ in dry $\mathrm{CH}_{2} \mathrm{Cl}_{2}(10 \mathrm{~mL})$ was added 2 ketobutyric acid $(52.0 \mathrm{mg}, 0.510 \mathrm{mmol})$ in dry $\mathrm{CH}_{2} \mathrm{Cl}_{2}(5$ $\mathrm{mL}$ ). The solution was stirred at room temperature for $0.5 \mathrm{~h}$. To this mixture were added HPR (100 $\mathrm{mg}, 0.255 \mathrm{mmol}$ ) in dry DMF ( $2 \mathrm{~mL})$ and DMAP (cat.) and the mixture was stirred for $2-3 \mathrm{~h}$. The reaction was quenched with $\mathrm{NH}_{4} \mathrm{Cl}$ $(a q)$, and the mixture was subjected to extraction with EtOAc $(2 \times 5 \mathrm{~mL})$. The extracts were washed with $\mathrm{H}_{2} \mathrm{O}$ and brine, dried $\left(\mathrm{Na}_{2} \mathrm{SO}_{4}\right)$. and concentration. The residue was purified by column chromatography using hexane/EtOAc (3/1) as the eluent to give HPR-2-ketobutyrate (60 $\mathrm{mg} .50 \%$ ) as a yellow solid.

${ }^{1} \mathrm{H}$ NMR (200 MHz. $\mathrm{CDCl}_{3}$ ): $\delta 7.60$ (d. $2 \mathrm{H} . J=8.9 \mathrm{~Hz}$ ). $7.13($ d. $2 \mathrm{H} . J=8.9 \mathrm{~Hz}), 7.01$ (dd. $1 \mathrm{H}, J=14.8 .11 .4 \mathrm{~Hz}$ ). 6.09-6.32 (m. $4 \mathrm{H}), 5.78$ (s. $1 \mathrm{H}), 2.98$ (m. $2 \mathrm{H}) .2 .42$ (s. $3 \mathrm{H}$ ). 2.01 (br s. $5 \mathrm{H}), 1.72(\mathrm{~s}, 3 \mathrm{H}), 1.56-1.68(\mathrm{~m}, 2 \mathrm{H}), 1.4+1.49$ (m. $2 \mathrm{H}$ ). 1.20 (t. $3 \mathrm{H}, J=7.2 \mathrm{~Hz}$ ), 1.03 (s. $6 \mathrm{H}$ ).

${ }^{13} \mathrm{C}$ NMR $\left(100 \mathrm{MHz} . \mathrm{CDCl}_{3}\right): \delta 194.14 .165 .05,159.32$. $153.90,151.30,145.89 .139 .55,137.67,137.21,136.74$. $136.66,135.06,130.75,129.99,129.43,128.66,125.45$. 121.46. 120.58. 39.55, 34.24, 33.08. 33.02. 28.93, 21.74 . $19.87,13.72,12.90,11.17$

MS: $m z(\%)=69(93), 109(100), 119(65), 161(69), 202$ (42). $255(28), 391(55), 475\left(18 . \mathrm{M}^{-}\right)$.

$4-\{[(2 E, 4 E, 6 E, 8 E)-3,7-D i m e t h y]-9-(2,6,6$-trimethyl-1cyclohexenyl)-2,4,6,8-nonatetraenoyl]amino\} phenyl-2oxopropanoate(2). Yield: $52 \%$

${ }^{l} \mathrm{H}$ NMR (200 MHz. $\left.\mathrm{CDCl}_{3}\right): \delta 7.60($ d. $2 \mathrm{H} . J=8.9 \mathrm{~Hz})$. 7.13 (d. $2 \mathrm{H} . J=8.9 \mathrm{~Hz}$ ). 7.01 (dd. $1 \mathrm{H} . J=14.8 .11 .4 \mathrm{~Hz}$ ). 6.09-6.32 (m. 4H). 5.78 (s. 1H). 2.59 (s. 3H). 2.43 (s. $3 \mathrm{H}$ ). 2.01 (br s. $5 \mathrm{H}$ ). 1.72 (s. $3 \mathrm{H}), 1.56-1.68$ (m. $2 \mathrm{H}$ ) $.1 .44-1.49$ (m. 2H). 1.03 (s. 6H).

${ }^{13} \mathrm{C}$ NMR $\left(100 \mathrm{MHz}, \mathrm{CDCl}_{3}\right): \delta 191.03,165.15,151.30$. 145.86. 139.56 137.68. 137.21. 136.71, 135.98. 135.07, 130.76. 130.00 . 129.43, 128.67. 121.39, 120.75, 120.62, 39.55.34.24. $33.08,29.68,28.94,26.82 .21 .74,19.18,13.73 .12 .91$.

MS: $m z(\%)=69(100), 109(92), 149(95), 201(25) .255$
(128), $391(28), 461\left(16, \mathrm{M}^{+}\right)$.

$4-\{[(2 E, 4 E, 6 E, 8 E)-3,7-D i m e t h y l-9-(2,6,6-t r i m e t h y l-1-$ cyclohexenyl)-2,4,6,8-nonatetraenoyl]amino\}phenyl-3hydroxybutanoate (3). Yield: $62 \%$

${ }^{1} \mathrm{H}$ NMR $\left(200 \mathrm{MHz}, \mathrm{CDCl}_{3}\right): \delta 7.56$ (d. $\left.2 \mathrm{H} . J=8.7 \mathrm{~Hz}\right)$, 7.25 (br s. $\mathrm{HH}$ ). 6.93-7.06 (m, 3H). 6.09-6.32 (m, $4 \mathrm{H}), 5.78$ (s, lH). 4.31-4.40 (m. lH), 2.68-2.81 (m. $2 \mathrm{H}), 2.43$ (s. $3 \mathrm{H})$, 2.01 (br s, 5H), 1.72 (s. $3 \mathrm{H}$ ). 1.56-1.68 (m. $2 \mathrm{H}$ ), 1.44-1.49 (m. 3H), $1.20-1.38(\mathrm{~m}, 2 \mathrm{H}) .1 .03(\mathrm{~s}, 6 \mathrm{H})$.

${ }^{13} \mathrm{C}$ NMR $\left(100 \mathrm{MHz}, \mathrm{CDCl}_{2}\right): \delta 171.20,148.29 .138 .39$, $137.20,136.80 .136 .63,135.27,129.34,129.17 .127 .81$. $121.86,121.23,121.08,120.65,120.58$. 119.91. 63.67 , $43.23,33.76,32.59,28.50,22.62 .22 .56 .21 .29 .19 .18$. $18.72,13.15 .12 .43$.

MS: $m z(\%)=58(100), 69(77), 109(77), 119(64), 161$ (56). $201(33), 255(32), 39 \mathrm{l}(23), 477\left(28, \mathrm{M}^{-}\right)$.

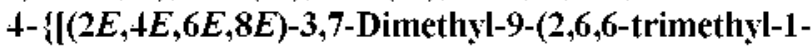
cyclohexenyl)-2,4,6,8-nonatetraenoyl]amino\}phenyl-2(dimethylamino)acetate (4). Yield: $75 \%$

${ }^{1} \mathrm{H}$ NMR $\left(200 \mathrm{MHz}, \mathrm{CDCl}_{3}\right.$ ): $\delta 7.55$ (d. $2 \mathrm{H} . J=8.7 \mathrm{~Hz}$ ), $7.05($ d. $2 \mathrm{H}, J=8.7 \mathrm{~Hz}), 7.0 \mathrm{l}($ dd. $1 \mathrm{H}, J=14.8,11.4 \mathrm{~Hz}$ ), $6.09-6.3 \mathrm{l}(\mathrm{m} .4 \mathrm{H}), 5.78(\mathrm{~s}, \mathrm{lH}) .2 .44(\mathrm{~s} .6 \mathrm{H}) .2 .42(\mathrm{~s} .3 \mathrm{H})$, $2.0 \mathrm{l}(\mathrm{br} \mathrm{s}, 5 \mathrm{H}), 1.72$ (s. $3 \mathrm{H}$ ). $1.56-1.68$ (m. $2 \mathrm{H}$ ), 1.44-1.49 (m. $2 \mathrm{H}), 1.03$ (s. $6 \mathrm{H})$.

${ }^{13} \mathrm{C}$ NMR $\left(100 \mathrm{MHz}, \mathrm{CDCl}_{3}\right): \delta 169.31,150.78 .146 .16$, $139.29,137.65 .137 .23,136.10,135.22,130.46,130.13$. $129.89,129.47 .128 .49,122.14,121.76,121.09 .120 .63$. $115.74,60.19,45.20 .39 .52,34.20,33.05 .29 .65,28.91$. $21.73,19.71 .13 .66,12.87$

MS: $m z(\%)=58(100), 69(6) .149(8), 476\left(73, \mathrm{M}^{+}\right)$.

4-(4-\{[(2E,4E,6E,8E)-3,7-Dimethyl-9-(2,6,6-trimethyl1-cyclohexenyl)-2,4,6,8-nonatetraenoyl]amino\}phenoxy)4-oxobutanoic acid (5). Yield: $72 \%$

${ }^{1} \mathrm{H}$ NMR (200 MHz. $\mathrm{CDCl}_{3}$ ): $\delta 7.40-7.50$ (br s. $2 \mathrm{H}$ ). 6.99 $7.04(\mathrm{~m} .3 \mathrm{H}), 6.09-6.30(\mathrm{~m}, 4 \mathrm{H}) .5 .79(\mathrm{~s}, \mathrm{lH}) .4 .40-4.55$ (br s. $\mathrm{lH}$ ). $2.82 .2 .84(\mathrm{~m}, 4 \mathrm{H}), 2.40$ (s. $3 \mathrm{H}), 2.00(\mathrm{br} \mathrm{s}, 5 \mathrm{H})$, $1.72(\mathrm{~s} .3 \mathrm{H}), 1.56-1.68(\mathrm{~m}, 2 \mathrm{H}) .1 .44-1.49(\mathrm{~m}, 2 \mathrm{H}) .1 .03(\mathrm{~s}$, $6 \mathrm{H})$.

${ }^{13} \mathrm{C}$ NMR (100 MHz, $\left.\mathrm{CDCl}_{2}\right): \delta 174.52,171.23,159.32$ 146.16. 139.55. 137.71, 137.60. 137.19. 136.09, 135.57. 130.75. 130.00. 129.80. 129.53, 128.21. 122.11. 121.65, 120.36. 39.89. $34.14 .33 .01,29.32,29.06,28.97,28.87,21.67,19.12,13.55$. 12.81 .

MS: $m z(\%)=58(100) .69(22) .105(9), 135(8), 161(8)$. $391(7) .491\left(35 . \mathrm{M}^{-}\right)$.

General procedure III; $4-\{[(2 E, 4 E, 6 E, 8 E)-3,7$-Dimethy9-(2,6,6-trimethyl-1-cyclohexenyl)-2,4,6,8-nonatetraenoyl]amino:phenyl-1-propanesulfonate (6). To a cold solution of HPR (100 mg. $0.255 \mathrm{mmol})$ in dry $\mathrm{CH}_{2} \mathrm{Cl}_{2}(10 \mathrm{~mL})$ and $N$-methy lmorpholine $(0.034 \mathrm{~mL}, 0.306 \mathrm{mmol})$ was added 1propane sulfonyl chloride $(0.034 \mathrm{~mL}, 0.306 \mathrm{mmol})$. After being stirred for $30 \mathrm{~min}$. the mixture was further stirred for 2 $\mathrm{h}$ at room temperature. Saturated aqueous $\mathrm{NH}_{4} \mathrm{Cl}$ was added into the mixture. and the aqueous layer was extracted with EtOAc $(2 \times 5 \mathrm{~mL})$. The combined organic layers were washed with $\mathrm{H}_{2} \mathrm{O}$ and brine. dried $\left(\mathrm{Na}_{2} \mathrm{SO}_{4}\right)$, and concentration. The residue was purified by colunın chromatography (EtOAc/ 
Hexane $=1: 3$ ) to provide HPR-propyl sulfonate $(95 \mathrm{mg}$. $75 \%$ ) as a yellow solid.

${ }^{1} \mathrm{H}$ NMR (200 MHz. $\mathrm{CDCl}_{3}$ ): $\delta 7.59$ (d. $2 \mathrm{H} . J=8.9 \mathrm{~Hz}$ ). 7.18-7.27 (n. 3H), $7.0 \mathrm{l}$ (dd. $1 \mathrm{H}, J=14.8 .11 .4 \mathrm{~Hz}) .6 .09-$ $6.31(\mathrm{ml} .4 \mathrm{H}) .5 .78(\mathrm{~s}, 1 \mathrm{H}), 3.22(\mathrm{~d} .2 \mathrm{H}, J=7.8 \mathrm{~Hz}) .2 .4 \mathrm{l}(\mathrm{s}$. $3 \mathrm{H}), 1.95-2.06(\mathrm{~m} .7 \mathrm{H}), 1.72(\mathrm{~s}, 3 \mathrm{H}), 1.56-1.68(\mathrm{~m}, 2 \mathrm{H})$. $1.44-1.49(\mathrm{~m} .2 \mathrm{H}), 1.10(\mathrm{t} .3 \mathrm{H} . J=7.3 \mathrm{~Hz}) .1 .03(\mathrm{~s}, 6 \mathrm{H})$.

${ }^{13} \mathrm{C}$ NMR $\left(100 \mathrm{MHz} . \mathrm{CDCl}_{3}\right): \delta 165.30 .151 .24,144.66$. $139.52,137.64,137.41 .137 .17,135.07 .130 .72,129.97$, 129.41. 128.64, 122.47, 120.90. 120.77, 51.87. 39.54. 34.21. $33.06,28.91,21.70,19.16,17.26,13.72,12.87 .12 .80$.

MS: $m z(\%)=108(100), 123(12), 215(13), 497\left(50 . \mathrm{M}^{-}\right)$. 4- $\{[(2 E, 4 E, 6 E, 8 E)$-3,7-Dimethyl-9-(2,6,6-trimethyl1-cyclohexenyl)-2,4,6,8-nonatetraenoyl] amino; phenyl1-butanesulfonate (7). Yield: $73 \%$

${ }^{1} \mathrm{H}$ NMR (200 MHz. $\mathrm{CDCl}_{3}$ ): $\delta 7.57$ (d. $2 \mathrm{H} . J=8.9 \mathrm{~Hz}$ ). 7.53 (br s, $1 \mathrm{H}$ ), 7.18 (d. $2 \mathrm{H}, J=8.9 \mathrm{~Hz}$ ). $7.0 \mathrm{l}$ (dd. $1 \mathrm{H} . J=$ $14.8,11.4 \mathrm{~Hz}) .6 .09-6.3 \mathrm{l}(\mathrm{m}, 4 \mathrm{H}), 5.80(\mathrm{~s}, 1 \mathrm{H}), 3.22$ (d. $2 \mathrm{H}$. $J=7.8 \mathrm{~Hz}) .2 .4 \mathrm{l}(\mathrm{s}, 3 \mathrm{H}) .1 .87-2.0 \mathrm{l}(\mathrm{m} .7 \mathrm{H}) .1 .72(\mathrm{~s}, 3 \mathrm{H})$. 1.56-1.68 (m. 2H). 1.44-1.49 (m, 4H), 1.01 (s, 6H). 0.97 (t. $3 \mathrm{H}, J=7.3 \mathrm{~Hz})$.

${ }^{13} \mathrm{C}$ NMR (100 MHz. $\left.\mathrm{CDCl}_{3}\right): \delta 165.23 .151 .38,144.71$. $139.58,137.66,137.37,137.19,135.05,130.80,130.00$. 129.41. 128.69, 122.52, 120.86. 120.70, 49.95. 39.54. 34.23. $33.07,28.92 .25 .36,21.73,21.39 .19 .17,13.73,13.45$. 12.89 .

MS: $m z(\%)=108(100), 136(12), 204(10), 511\left(70 . \mathrm{M}^{-}\right)$.

General procedure IV; $+\{[(2 E, 4 E, 6 E, 8 E)-3,7$-dimethyl-9(2,6,6-trimethyl-1-cycloherenyl)-2,4,6,8-nonatetraenoyl]aminosphenyl propyl hydrogen phosphate (8). To a solution propanol $(0.8 \mathrm{~mL}, 0.010 \mathrm{~mol})$ in dry $\mathrm{CH}_{2} \mathrm{Cl}_{2}(10$ $\mathrm{mL}$ ) was added $\mathrm{POCl}_{3}(3.0 \mathrm{~mL}, 0.032 \mathrm{~mol})$ dropwise at 0 ${ }^{\circ} \mathrm{C}$. After being stirred at $0{ }^{\circ} \mathrm{C}$ for $\mathrm{I} \mathrm{h}$. the mixture was refluxed for $5 \mathrm{~h}$, followed by vacuum distillation (bp 38-42 ${ }^{\circ} \mathrm{C} / 0.2$ torr) to afford propyl dichlorophosphate.

To a cooled solution of propyl dichlorophosphate $(1 e q$.$) in$ dry $\mathrm{CH}_{2} \mathrm{Cl}_{2}(5 \mathrm{~mL})$ were added $\mathrm{HPR}(0.1 \mathrm{~g} .0 .33 \mathrm{mmol})$ in dry $\mathrm{CH}_{2} \mathrm{Cl}_{2}(5 \mathrm{~mL})$ and triethylamine $(0.11 \mathrm{~mL}, 0.83 \mathrm{mmol})$. After being stirred for $1 \mathrm{~h}$. $\mathrm{H}_{2} \mathrm{O}(2 \mathrm{~mL})$ was added into the mixture. The mixture was stirred for $2 \mathrm{~h}$ at room temperature and quenched with $\mathrm{NH}_{4} \mathrm{Cl}(\mathrm{aq}$.). The aqueous layer was extracted with $\mathrm{CH}_{2} \mathrm{Cl}_{2}(2 \times)$, and the combined organic layers were washed with $\mathrm{H}_{2} \mathrm{O}$ and brine. dried $\left(\mathrm{Na}_{2} \mathrm{SO}_{4}\right)$, and concentrated. The residue was purified by column cluromatography $(\mathrm{EtOAc} / \mathrm{Hexane}=1: 2$ ) to afford HPR-propyl phosphate $(96 \mathrm{mg} .73 \%)$ as a yellow solid.

${ }^{l} \mathrm{H}$ NMR (400 MHz. $\left.\mathrm{CDCl}_{3}\right): \delta 7.56$ (d. $2 \mathrm{H} . J=7.8 \mathrm{~Hz}$ ). 7.39 (br s. $1 \mathrm{H}$ ) .7 .15 (d. $2 \mathrm{H} . J=8.7 \mathrm{~Hz}$ ). 7.01 (dd. $1 \mathrm{H} . J=$ 14.8. $11.4 \mathrm{~Hz}) .6 .12-6.30(\mathrm{~m} .4 \mathrm{H}) .5 .79(\mathrm{~s} .1 \mathrm{H}) .4 .28(\mathrm{~m}$. $2 \mathrm{H}) .2 .41$ (s. 3H). 2.02 (br s. $5 \mathrm{H}$ ). 1.79-1.83 (m. 2H). 1.72 (s. $3 \mathrm{H}) .1 .60-1.63(\mathrm{~m} .2 \mathrm{H}), 1.45-1.48(\mathrm{~m} .2 \mathrm{H}), 1.02(\mathrm{~s} .6 \mathrm{H})$. $0.96-1.00(\mathrm{~m}, 3 \mathrm{H})$

${ }^{13} \mathrm{C}$ NMR $\left(100 \mathrm{MHz}, \mathrm{CDCl}_{3}\right): \delta 166.19 .151 .73,145.59$. $139.51,137.69,137.22,136.89,135.13,130.70,129.99$. 129.44. 128.65, 121.12, 120.85, 120.80, 72.15. 72.08, 39.58 . $34.24,33.09,28.94,23.29,21.74,19.20,13.72$. 12.91 .

MS: $m z(\%)=108(100) .119(41) .159(71), 202(25)$.
$225(18), 39 \mathrm{l}(38), 513\left(66, \mathrm{M}^{+}\right)$.

Butyl-(4-\{[(2,4,6E,8E)-3,7-dimethyl-9-(2,6,6-trimethyl1-cyclohexenyl)-2,4,6,8-nonatetraenoyl]amino\}phenyl) hydrogen phosphate (9). Yield: $71 \%$

${ }^{1} \mathrm{H}$ NMR (400 MHz. $\left.\mathrm{CDCl}_{3}\right): \delta 7.89$ (br s, lH). $7.55(\mathrm{~d}$, $2 \mathrm{H} . J=7.8 \mathrm{~Hz}$ ). 7.15 (d, $2 \mathrm{H} . J=8.7 \mathrm{~Hz}$ ). 6.99 (dd. $1 \mathrm{H} . J=$ 14.8. $11.4 \mathrm{~Hz}$ ), 6.12-6.29 (m. $4 \mathrm{H}) .5 .84$ (s. $1 \mathrm{H}) .4 .32$ (m. $2 \mathrm{H}) .2 .4 \mathrm{l}(\mathrm{s} .3 \mathrm{H}), 2.02(\mathrm{br} \mathrm{s}, 5 \mathrm{H}), 1.74-1.8 \mathrm{l}(\mathrm{m} .2 \mathrm{H}), 1.72(\mathrm{~s}$, $3 \mathrm{H}$ ). $1.60-1.63$ (m. $2 \mathrm{H}), 1.41-1.48(\mathrm{~m}, 4 \mathrm{H}) .1 .03$ (s. $6 \mathrm{H})$, $0.97(\mathrm{t}, 3 \mathrm{H}, J=7.3 \mathrm{~Hz})$,

${ }^{13} \mathrm{C}$ NMR $\left(100 \mathrm{MHz}, \mathrm{CDCl}_{2}\right): \delta 165.29,150.83 .144 .89$, $139.29,137.66 .137 .21,136.69,135.30,130.43 .129 .92$. 129.48, 128.57, 120.99. 120.69. 120.64. 70.52, 70.44, 39.54 . $34.22,33.06,31.74,31.67,28.91$. 21.71. 19.17. 18.53. 13.67, 13.43. 12.87 .

MS: $m z(\%)=69(46), 108(83) .119(30), 201(20) .255$ (15), $39 \mathrm{l}(14), 527\left(42 . \mathrm{M}^{-}\right)$.

Cell proliferation assays. Effects of 4-HPR derivatives on the proliferation of cervical cancer cell were determined using MTT assay kit (Sigma Co.. St. Louis, MO) according to the manufacturers manual. Cells were grown in 96 wellmicrotiter plates starting at an initial density of $3 \times 10^{3} \mathrm{cells} /$ $100 \mathrm{~mL} \mathrm{medium} / \mathrm{well}$. After $12 \mathrm{~h}$ from seeding. indicated concentrations of derivatives were added to the medium. and the cells were further grown for $48 \mathrm{~h}$. The use of $0.01 \%$ DMSO as control of RA. did not affect proliferation of cells tested. After $4 \mathrm{~h}$ incubation with MTT reagent. the media was removed. DMSO $(150 \mathrm{~mL})$ was added to precipitate and the absorbance at $550 \mathrm{~nm}$ was measured using Spectra MAX 250 microplate spectrophotometer (Molecular Devices, Sunnyvale, CA). The results were expressed as mean values of the absorbances of at least four wells.

Acknowledgment. This study was supported by a grant of the Korea Health Ministry of Health \& Welfare, Republic of Korea (02-PII-PGI l-VN01-SV01-0019).

\section{References}

1. Tallman. M. S.: Wiernik. P. H. J. Chn. Phomacol 1992. 32.868.

2. Vinienti, M. P.; Clark. I. M.: Brinkerhoff, C. E. Arthritis Rhetntatoidism 1994. 37, 1125.

3. Rottman. J. N.: Widom, R. L; Nadal-Ginard, B; Mahdavi, V: Karathanansis. S. K. Mol. Cell Biol. 1991. 3814.

4. Yang. Y: Vacchio. M. S.: Aswell. T. D. Proc. Katl. Acad. Sci. U.S.A. 1991. 90.6170.

5. Sporn. M. B.: Roberts. A. B.: Goodman, O. S. The Rethoids: Biolog: Chemistry and Medicine. 2nd ed.: Raven Press: New York. 1994.

6. Smith. M. A.: Parkinson. D. R.: Cheson. B. D.: Friedman. M. A.J. Clin. Oncol 1992. 10.839.

7. Kenel. M. F.: Krayer. J. H.: Merz. E. A.: Pritchard. I. F. Teratog. Carcing. Mutag, 1988, 8, 1 .

8. Abou-Issa. H.; Curley. R. W. Jr.: Panigot, M. J.; Tanagho, S. N.: Sidhu. B. S.: Alshafie. G. A. Anticancer Res. 1997, 17.3335.

9. Veronesi. U.: DePalo. G.: Marubini. E.: Costa. A.: Fomelli. F.: Mariani. L.: Rosselli Del Turco. M.: Gaetana Di Mauro. M.: Grazia Muraca. M.: Del Vecehio. M.: Pinto. C.: D'Aiuto. G.: Boni. C.; Campa. T; Magni, A.: Miceli. R.; Perloff. M.: Malone. W. F.: Sporn, M. B. J. Natl Cancer Inst. 1999. 91, 1847.

10. Bhatnagar. R.: Abou-Issa, H.; Curley, R. W.: Koolemans-Beynen. 
A.: Moeschberger. M. L.: Webb. T. E. Biochem. Phamacol. 1991. 41. 1471 .

11. Um. S. J.: Lee. S. Y: Kim. E. J.: Han. H. S.: Koh. Y. M.: Hong. K. J.: Sin. H. S.: Park. J. S. Cancer Letter 2001. 174. 127.

12. Delia, D.: Aiello. A.: Lombardi. L.: Pelicei. P. G.: Grignani, F: Formelli. F.: Menard, S.: Costa. A.: Veronesi, U.: Pierotti, M. A.
Cancer Res. 1993, 53,6036.

13. Abou-Issa. H.: Curley. R. W: Panigot. M. J.: Wilcox. K. A.: Webb. T. E. Anticancer Res. 1993. 13. 1431.

14. Maryanoff. C. A. U.S. Patent 5399757. 1995.

15. Sangmam, C.; Winnum, J.-Y.: Lucas. M.: Montero. J.-L.: Chavis, C. Synth. Commu 1998. 28. 2945. 